\title{
Bourgain discretization using Lebesgue-Bochner spaces
}

\author{
Mikhail I. Ostrovskii and Beata Randrianantoanina
}

This paper is dedicated to the memory of our friend Joe Diestel (1943-2017). Lebesgue-Bochner spaces were one of the main passions of Joe. He started to work in this direction in his Ph.D. thesis [Die68], and devoted to Lebesgue-Bochner spaces a large part of his most popular, classical, Dunford-Schwartz-style monograph [DU77], joint with Jerry Uhl.

\begin{abstract}
We study the Lebesgue-Bochner discretization property of Banach spaces $Y$, which ensures that the Bourgain's discretization modulus for $Y$ has a good lower estimate. We prove that there exist spaces that do not have the Lebesgue-Bochner discretization property, and we give a class of examples of spaces that enjoy this property.
\end{abstract}

2010 Mathematics Subject Classification. Primary: 46B85; Secondary: 46B06, 46B07, 46E40.

Keywords. Bourgain discretization theorem, distortion of an embedding, Lebesgue-Bochner space

\section{Introduction}

We denote by $c_{Y}(X)$ the greatest lower bound of distortions of bilipschitz embeddings of a metric space $\left(X, d_{X}\right)$ into a metric space $\left(Y, d_{Y}\right)$, that is, the greatest lower bound of the numbers $C$ for which there is a map $f: X \rightarrow Y$ and a real number $r>0$ such that

$$
\forall u, v \in X \quad r d_{X}(u, v) \leq d_{Y}(f(u), f(v)) \leq r C d_{X}(u, v) .
$$

See [Mat02, Nao18, and [Ost13] for background on this notion. Let $X$ be a finitedimensional Banach space and $Y$ be an infinite-dimensional Banach space.

Definition 1.1. For $\varepsilon \in(0,1)$ let $\delta_{X \hookrightarrow Y}(\varepsilon)$ be the supremum of those $\delta \in(0,1)$ for which every $\delta$-net $\mathcal{N}_{\delta}$ in $B_{X}$ satisfies $c_{Y}\left(\mathcal{N}_{\delta}\right) \geq(1-\varepsilon) c_{Y}(X)$. The function $\delta_{X \hookrightarrow Y}(\varepsilon)$ is called the discretization modulus for embeddings of $X$ into $Y$.

It is not immediate that the discretization modulus is defined for any $\varepsilon \in(0,1)$, but this can be derived using the argument of [Rib76] and HM82 (see GNS12, Introduction]). Giving a new proof of the Ribe theorem Rib76] Bourgain proved the following remarkable result Bou87] (we state it in a stronger form which was proved in GNS12]): 
Theorem 1.2 (Bourgain's discretization theorem). There exists $C \in(0, \infty)$ such that for every two Banach spaces $X, Y$ with $\operatorname{dim} X=n<\infty$ and $\operatorname{dim} Y=\infty$, and every $\varepsilon \in(0,1)$, we have

$$
\delta_{X \hookrightarrow Y}(\varepsilon) \geq e^{-\left(c_{Y}(X) / \varepsilon\right)^{C n}} .
$$

Bourgain's discretization theorem and the described below result of [GNS12] on improved estimates in the case of $L_{p}$ spaces have important consequences for quantitative estimates of $L_{1}$-distortion of the metric space consisting of finite subsets (of equal cardinality) in the plane with the minimum weight matching distance, see [NS07, Theorem 1.2].

The proof of Bourgain's discretization theorem was clarified and simplified in [Beg99 and GNS12 (see also its presentation in Ost13, Section 9.2]). Different approaches to proving Bourgain's discretization theorem in special cases were found in [LN13], HLN16], and [HN16+. However these approaches do not improve the order of estimates for the discretization modulus.

On the other hand the paper GNS12 contains a proof with much better estimates in the case where $Y=L_{p}$. The approach of [GNS12] is based on the following result (whose proof uses methods of [JMS09]; origins of this approach can be found in GK03).

Theorem 1.3 (GNS12, Theorem 1.3]). There exists a universal constant $\kappa \in(0, \infty)$ with the following property. Assume that $\delta, \varepsilon \in(0,1)$ and $D \in[1, \infty)$ satisfy $\delta \leq$ $\kappa \varepsilon^{2} /\left(n^{2} D\right)$. Let $X, Y$ be Banach spaces with $\operatorname{dim} X=n<\infty$, and let $\mathcal{N}_{\delta}$ be a $\delta$-net in $B_{X}$. Assume that $c_{Y}\left(\mathcal{N}_{\delta}\right) \leq D$. Then there exists a separable probability space $(\Omega, \nu)$, a finite dimensional linear subspace $Z \subseteq Y$, and a linear operator $T: X \rightarrow L_{\infty}(\nu, Z)$ satisfying

$$
\forall x \in X, \quad \frac{1-\varepsilon}{D}\|x\|_{X} \leq\|T x\|_{L_{1}(\nu, Z)} \leq\|T x\|_{L_{\infty}(\nu, Z)} \leq(1+\varepsilon)\|x\|_{X} .
$$

As is noted in GNS12, since $(\Omega, \nu)$ is a probability measure, we have

$$
\|\cdot\|_{L_{1}(\nu, Z)} \leq\|\cdot\|_{L_{p}(\nu, Z)} \leq\|\cdot\|_{L_{\infty}(\nu, Z)}
$$

for every $p \in[1, \infty]$, therefore (2) implies that $X$ admits an embedding into $L_{p}(\nu, Z)$ with distortion $\leq \frac{D(1+\varepsilon)}{1-\varepsilon}$. Since, by the well-known Carathéodory theorem, $L_{p}\left(\nu, L_{p}\right)$ is isometric to $L_{p}$ (see [Lac74, $\left.\S 14\right]$ ) we get that if $Z$ is a subspace of $L_{p}$, then $L_{p}(\nu, Z)$ is also a subspace of $L_{p}$, and, as explained in GNS12, it follows that the Bourgain's discretization modulus for the case of $Y=L_{p}$ satisfies a much better estimate

$$
\delta_{X \hookrightarrow L_{p}}(\varepsilon) \geq \frac{\kappa \varepsilon^{2}}{n^{5 / 2}}
$$

(since for all spaces $X, Y$ and all $\delta>0, c_{Y}\left(\mathcal{N}_{\delta}\right) \leq \sqrt{n}$, see [GNS12]).

To generalize this approach to a wider class of spaces it is natural to introduce the following definition. 
Definition 1.4. We say that a Banach space $Y$ has the Lebesgue-Bochner discretization property if for any separable probability measure $\mu$, there exists a function $f:[1, \infty) \rightarrow[1, \infty)$ so that for any $C \geq 1$ and any finite dimensional subspace $Z \subset Y$, if $W$ is any finite-dimensional subspace of $L_{\infty}(\mu, Z)$ such that for all $w \in W$

$$
\|w\|_{L_{\infty}(\mu, Z)} \leq C\|w\|_{L_{1}(\mu, Z)},
$$

then $W$ is $f(C)$-embeddable into $Y$.

The following is a corollary of Theorem 1.3 .

Corollary 1.5. Let $Y$ be a Banach space with the Lebesgue-Bochner discretization property, $\delta \leq \kappa \varepsilon^{2} /\left(n^{5 / 2}\right)$, where $\kappa$ is the constant of Theorem 1.3, and $\mathcal{N}_{\delta}$ be a $\delta$-net in an $n$-dimensional Banach space $X$. Then

$$
c_{Y}(X) \leq g\left(\frac{1+\varepsilon}{1-\varepsilon} c_{Y}\left(\mathcal{N}_{\delta}\right)\right),
$$

where $g(t):=t f(t)$ and $f$ is the function of Definition 1.4. Thus if, for an increasing function $g$, we define $\delta_{X \hookrightarrow Y}^{g}(\varepsilon)$ as the supremum of $\delta$ so that (4) is satisfied for all $\delta$-nets $\mathcal{N}_{\delta}$ of $B_{X}$, we have that $\delta_{X \hookrightarrow Y}^{g}(\varepsilon) \geq \kappa \varepsilon^{2} /\left(n^{5 / 2}\right)$.

Proof. By Theorem 1.3, there exists a finite dimensional subspace $Z \subset Y$ and a finite-dimensional subspace $W \subset L_{\infty}(\nu, Z)$ (the image of the operator $T$ ) so that $W$ satisfies (3) with $C=\frac{1+\varepsilon}{1-\varepsilon} c_{Y}\left(\mathcal{N}_{\delta}\right)$. Thus by the Lebesgue-Bochner discretization property of $Y, c_{Y}(W) \leq f\left(\frac{1+\varepsilon}{1-\varepsilon} c_{Y}\left(\mathcal{N}_{\delta}\right)\right)$, and we obtain

$$
c_{Y}(X) \leq \frac{1+\varepsilon}{1-\varepsilon} c_{Y}\left(\mathcal{N}_{\delta}\right) f\left(\frac{1+\varepsilon}{1-\varepsilon} c_{Y}\left(\mathcal{N}_{\delta}\right)\right) .
$$

Problem 1. Characterize Banach spaces with the Lebesgue-Bochner discretization property.

At the meeting of the Simons Foundation (New York City, February 20, 2015) Assaf Naor mentioned that at that time no examples of Banach spaces which do not have the Lebesgue-Bochner discretization property were known although people who were working on this (Assaf Naor and Gideon Schechtman) believed that such examples should exist.

We note that the based on the Fubini and Carathéodory theorems argument showing that $L_{p}\left(L_{p}\right)$ is isometric to $L_{p}$ (for suitable measure spaces) fails for other functions spaces even in a certain 'isomorphic' form (see [BBS02, Appendix]). For some spaces a very strong opposite of the situation in the $L_{p}$-case happens: Raynaud Ray89 proved that when $L_{\varphi}([0,1], \mu)$ is an Orlicz space that is not isomorphic to some $L_{p}$ and does not contain $c_{0}$ or $\ell_{1}$, then, for any $r \in[1, \infty)$ the space $\ell_{r}\left(L_{\varphi}\right)$ (and thus also $L_{\varphi}\left([0,1], \mu, L_{\varphi}\right)$ ) not only does not embed in $L_{\varphi}([0,1], \mu)$, but is not even crudely finitely representable in it. 
In general, if $E$ is a Banach function space on a measure space $(\Omega, \mu)$, the structure of the $E$-valued Bochner space $E(\Omega, \mu, E)$ can be very different from the structure of the space $E$, see [Rea90], BBS02], [FPP08]. We refer the reader to [BBS02 for a detailed discussion and history of related results.

In this paper we show (Proposition 2.2) that there is a class of Banach spaces which do not have the Lebesgue-Bochner discretization property and observe that this class contains the space constructed by Figiel [Fig72].

We also find some examples, besides $L_{p}$, of Banach spaces that have the LebesgueBochner discretization property. An easy observation is that the Lebesgue-Bochner spaces $L_{p}(E)$, where $E$ is any Banach space, have the Lebesgue-Bochner discretization property. It is interesting that even the finite direct sums of such spaces have the Lebesgue-Bochner discretization property, see Proposition 3.1. We would like to mention that many well-known and important spaces are of the form $L_{p}(E)$. In particular, the mixed norm Lebesgue spaces $L^{P}$ introduced in BP61 are such and thus have the Lebesgue-Bochner discretization property. For $P=\left(p_{1}, \ldots, p_{m}\right) \in[1, \infty)^{m}$, the space $L^{P}$ consists of measurable functions $f$ on $\Omega=\prod_{i=1}^{m}\left(\Omega_{j}, \mu_{j}\right)$, the norm defined by

$$
\|f\|_{P}:=\left(\int \ldots\left(\int\left(\int\left|f\left(t_{1}, \ldots, t_{n}\right)\right|^{p_{1}} d \mu_{1}\right)^{p_{2} / p_{1}} d \mu_{2}\right)^{p_{3} / p_{2}} \ldots d \mu_{m}\right)^{1 / p_{m}} .
$$

Mixed norm spaces of this type arise naturally in harmonic and functional analysis. Such norms (and their generalizations that use other function space norms in place of the $L_{p_{j}}$-norms) are used for example to study Fourier and Sobolev inequalities and embeddings of Sobolev spaces. The properties and applications of mixed norm spaces are extensively studied in the literature, see e.g. [GS16, CS16, DPS10] and their references.

\section{Finitely squarable Banach spaces}

Definition 2.1. An infinite-dimensional Banach space $Y$ is called finitely squarable if there exists a constant $C$ such that for every finite-dimensional subspace $Z \subset Y$ the direct sum $Z \oplus_{\infty} Z$ admits a linear embedding into $Y$ with distortion bounded by $C$.

The first examples of Banach spaces which are not finitely squarable were constructed by Figiel Fig72]. An easy observation is that a Banach space $Y$ which is isomorphic to $Y \oplus Y$, is finitely squarable. The converse it false. In fact, both of the earliest examples of Banach spaces which are not isomorphic to their squares, the James [Jam50] quasireflexive space $J$ [BP60] and $c\left(\omega_{1}\right)$ [Sem60] are finitely squarable, and for very simple reason: they have trivial cotype. For the James space this was proved in GJ73, for $c\left(\omega_{1}\right)$ this is obvious. Modern Banach space theory provides much more sophisticated examples of finitely squarable spaces which are not isomorphic to their squares, for example, the Argyros-Haydon space [AH11. 
Proposition 2.2. Any space which is not finitely squarable does not have the Lebesgue-Bochner discretization property.

Proof. Let $Z$ be a subspace of $Y$ for which $Z \oplus_{\infty} Z$ is "very far" from a subspace of $Y$.

We introduce the following subspace $W \subset L_{\infty}([0,1], Z)$ : it consists of all $Z$ valued functions which are constant on the first half and constant on the second half, but these constants can be different vectors of $Z$. It is clear that this space is isometric to $Z \oplus_{\infty} Z$. It is also clear that the $L_{1}([0,1], Z)$ norm on this subspace is 2-equivalent to the $L_{\infty}$-norm. The conclusion follows.

This proposition makes the following problem important:

Problem 2. Does there exist a finitely squarable space which does not have the Lebesgue-Bochner discretization property?

We conjecture that the answer to Problem 2 is positive.

\section{Examples of spaces with the Lebesgue-Bochner discretiza- tion property}

In this section we provide some examples of spaces having the Lebesgue-Bochner discretization property. In all proofs below we use the notation of Definition 1.4, that is: $Y$ is a Banach space, $C>0, Z \subset Y$ is a finite dimensional subspace of $Y$. Since we consider separable probability measures, by the Carathéodory theorem [Lac74 we may assume that $W$ is a finite-dimensional subspace of $L_{\infty}([0,1], \mu, Z)$, such that for all $w \in W$

$$
\frac{1}{C}\|w\|_{L_{\infty}([0,1], \mu, Z)} \leq\|w\|_{L_{1}([0,1], \mu, Z)} \leq\|w\|_{L_{\infty}([0,1], \mu, Z)} .
$$

Since $W$ is finite dimensional, for any $\varepsilon>0$, there exists a subspace $\tilde{W} \subseteq$ $L_{\infty}([0,1], \mu, Z)$ with Banach-Mazur distance from $W$ less than $1+\varepsilon$, so that $\tilde{W}$ is spanned by simple functions and all $w \in \tilde{W}$ satisfy (5) with $C$ replaced by $(1+\varepsilon) C$. Thus, without loss of generality, we may assume that $W$ is spanned by simple functions which are constant on elements $\left\{\Delta_{i}\right\}_{i=1}^{n}$ of some partition of $[0,1]$ into sets of measure $\frac{1}{n}$. Thus we can denote elements $w \in W$ as

$$
w=\left(w_{1}, \ldots, w_{n}\right),
$$

meaning that $w=\sum_{i=1}^{n} \mathbf{1}_{\Delta_{i}} \otimes w_{i}$. For all $w \in W$ we have $\|w\|_{L_{\infty}([0,1], \mu, Z)}=$ $\max _{1 \leq i \leq n}\left\|w_{i}\right\|_{Z}$, and for all $p, 1 \leq p<\infty$, we have

$$
\|w\|_{L_{p}([0,1], \mu, Z)}=\left(\frac{1}{n} \sum_{i=1}^{n}\left\|w_{i}\right\|_{Z}^{p}\right)^{\frac{1}{p}} .
$$


Given any $p \in[1, \infty], k \in \mathbb{N}$, and any Banach spaces $E_{1}, \ldots, E_{k}$, by $L_{p}^{k}\left(E_{1}, \ldots, E_{k}\right)$ we denote the Banach space of all $k$-tuples $\left(a_{1}, \ldots, a_{k}\right)$ such that $a_{j} \in E_{j}$ for all $j \in[k]$, endowed with the norm

$$
\begin{gathered}
\left\|\left(a_{1}, \ldots, a_{k}\right)\right\|_{L_{p}^{k}\left(E_{1}, \ldots, E_{k}\right)}:=\left(\frac{1}{k} \sum_{i=1}^{k}\left\|a_{i}\right\|_{Z}^{p}\right)^{\frac{1}{p}}, \text { if } p<\infty \\
\left\|\left(a_{1}, \ldots, a_{k}\right)\right\|_{L_{\infty}^{k}\left(E_{1}, \ldots, E_{k}\right)}:=\max _{1 \leq i \leq k}\left\|a_{i}\right\|_{Z} .
\end{gathered}
$$

If the spaces $E_{1}, \ldots, E_{k}$ are equal to the same space $E$, we denote $L_{p}^{k}\left(E_{1}, \ldots, E_{k}\right)$ by $L_{p}^{k}(E)$.

Proposition 3.1. Let $k \in \mathbb{N}, p, q_{1}, \ldots, q_{k} \geq 1, X_{1}, \ldots, X_{k}$ be any Banach spaces, and for each $j \in[k]$ let $\left(\Omega_{j}, \mu_{j}\right)$ be any nonatomic separable measure space, with finite or infinite measure, or $\Omega_{j}=\mathbb{N}$ and $\mu_{j}$ is the counting measure. Then the space

$$
Y=L_{p}^{k}\left(L_{q_{1}}\left(\Omega_{1}, \mu_{1}, X_{1}\right), L_{q_{2}}\left(\Omega_{2}, \mu_{2}, X_{2}\right), \ldots, L_{q_{k}}\left(\Omega_{k}, \mu_{k}, X_{k}\right)\right)
$$

has the Lebesgue-Bochner discretization property with $f(C) \leq k^{2-1 / p} C$.

Note that since the constant $f(C)$ in Definition 1.4 can depend on $k$, the fact that $Y$ is an $L_{p}^{k}$-sum is not essential, essential is the fact that $Y$ is a finite direct sum.

Proof of Proposition 3.1. To simplify notation we will omit the measure spaces when writing the symbol for a Lebesgue-Bochner space, i.e. we will write $L_{q_{j}}\left(X_{j}\right)$ instead of $L_{q_{j}}\left(\Omega_{j}, \mu_{j}, X_{j}\right)$ with the understanding that for all $j \in[k]$, the measure spaces are those fixed in the statement of the proposition.

Since for any $p \geq 1$, the space $L_{p}^{k}\left(L_{q_{1}}\left(X_{1}\right), L_{q_{2}}\left(X_{2}\right), \ldots, L_{q_{k}}\left(X_{k}\right)\right)$ is $k^{1-1 / p}$-isomorphic to $L_{1}^{k}\left(L_{q_{1}}\left(X_{1}\right), L_{q_{2}}\left(X_{2}\right), \ldots, L_{q_{k}}\left(X_{k}\right)\right)$, it is enough to prove that in the case where $p=1$ we have $f(C) \leq k C$. Note that if at least one of $q_{j}$ is equal to $\infty$, the space $Y$ has trivial cotype and thus has the Lebesgue-Bochner discretization property. In the following we assume that $q_{j}<\infty$ for all $j \in[k]$.

Using the discussion and notation preceding Proposition 3.1, we see that it suffices to prove that any subspace $W \subseteq L_{\infty}^{n}(Y)$ satisfying

$$
\forall w \in W \quad \frac{1}{C}\|w\|_{L_{\infty}^{n}(Y)} \leq\|w\|_{L_{1}^{n}(Y)}
$$

admits a $k C$-isomorphic embedding into $Y$.

Let $n \in \mathbb{N}, w=\left(w_{1}, \ldots, w_{n}\right) \in W \subseteq L_{\infty}^{n}(Y)$ and, for $i \in[n], w_{i}=\left(w_{i j}\right)_{j=1}^{k} \in$ $L_{1}^{k}\left(L_{q_{1}}\left(X_{1}\right), L_{q_{2}}\left(X_{2}\right), \ldots, L_{q_{k}}\left(X_{k}\right)\right)$, where, for all $i \in[n], j \in[k], w_{i j} \in L_{q_{j}}\left(X_{j}\right)$. We will define a map $\varphi$ from $L_{\infty}^{n}(Y)$ to $Y$ so that for all $w \in L_{\infty}^{n}(Y)$ we have

$$
\|\varphi(w)\|_{Y}=\frac{1}{k} \sum_{j=1}^{k}\left(\frac{1}{n} \sum_{i=1}^{n}\left\|w_{i j}\right\|_{q_{j}}^{q_{j}}\right)^{\frac{1}{q_{j}}} .
$$


For each $j \in[k]$, we select $n$ mutually disjoint subsets $\left\{\Omega_{j \nu}\right\}_{\nu=1}^{n}$ of $\Omega_{j}$ so that for each $\nu \in[n]$ there exists a constant $a_{j \nu}>0$ and a surjective isometry $T_{j \nu}$ : $L_{q_{j}}\left(\Omega_{j}, \mu_{j}\right) \rightarrow L_{q_{j}}\left(\Omega_{j \nu}, a_{j \nu} \mu_{j}\right)$. It is well-known that when $\left(\Omega_{j}, \mu_{j}\right)$ is nonatomic or equal to $\mathbb{N}$ with the counting measure then such choices are possible, and that the isometry $T_{j \nu}$ can be naturally extended to the isometry $\bar{T}_{j \nu}$ from the LebesgueBochner space $L_{q_{j}}\left(\Omega_{j}, \mu_{j}, X_{j}\right)$ onto $L_{q_{j}}\left(\Omega_{j \nu}, a_{j \nu} \mu_{j}, X_{j}\right)$, cf. e.g. [DU77.

We define the map $\varphi_{j}: L_{\infty}^{n}\left(L_{q_{j}}\left(X_{j}\right), \ldots, L_{q_{j}}\left(X_{j}\right)\right) \rightarrow L_{q_{j}}\left(X_{j}\right)$ by setting for all $\left(x_{1}, \ldots, x_{n}\right) \in L_{\infty}^{n}\left(L_{q_{j}}\left(X_{j}\right), \ldots, L_{q_{j}}\left(X_{j}\right)\right)$

$$
\varphi_{j}\left(x_{1}, \ldots, x_{n}\right):=\sum_{\nu=1}^{n}\left(\frac{a_{j \nu}}{n}\right)^{\frac{1}{q_{j}}} \bar{T}_{j \nu} x_{\nu} .
$$

Since the sets $\left\{\Omega_{j \nu}\right\}_{\nu=1}^{n}$ are mutually disjoint, we get

$$
\left\|\varphi_{j}\left(x_{1}, \ldots, x_{n}\right)\right\|_{q_{j}}=\left(\frac{1}{n} \sum_{\nu=1}^{n} a_{j \nu}\left\|\bar{T}_{j \nu} x_{\nu}\right\|_{q_{j}}^{q_{j}}\right)^{\frac{1}{q_{j}}}=\left(\frac{1}{n} \sum_{\nu=1}^{n}\left\|x_{\nu}\right\|_{q_{j}}^{q_{j}}\right)^{\frac{1}{q_{j}}} .
$$

Next, given $w=\left(w_{1}, \ldots, w_{n}\right) \in L_{\infty}^{n}(Y)$ where, for $i \in[n], w_{i}=\left(w_{i j}\right)_{j=1}^{k} \in Y=$ $L_{1}^{k}\left(L_{q_{1}}\left(X_{1}\right), L_{q_{2}}\left(X_{2}\right), \ldots, L_{q_{k}}\left(X_{k}\right)\right)$, we define $\varphi(w) \in Y$ by setting

$$
\varphi\left(\left(w_{i}\right)_{i=1}^{n}\right)=\left(\varphi_{j}\left(\left(w_{i j}\right)_{i=1}^{n}\right)\right)_{j=1}^{k} .
$$

By (8), equality (7) is satisfied.

We will show that for all $w \in W \subseteq L_{\infty}^{n}(Y)$, we have

$$
\|w\|_{L_{1}^{n}(Y)} \leq\|\varphi(w)\|_{Y} \leq k\|w\|_{L_{\infty}^{n}(Y)} .
$$

To prove the leftmost inequality we write

$$
\begin{aligned}
\|w\|_{L_{1}^{n}(Y)} & =\frac{1}{n} \sum_{i=1}^{n}\left\|w_{i}\right\|_{Y}=\frac{1}{n} \sum_{i=1}^{n}\left(\frac{1}{k} \sum_{j=1}^{k}\left\|w_{i j}\right\|_{q_{j}}\right) \\
& =\frac{1}{k} \sum_{j=1}^{k}\left(\frac{1}{n} \sum_{i=1}^{n}\left\|w_{i j}\right\|_{q_{j}}\right) \leq \frac{1}{k} \sum_{j=1}^{k}\left(\frac{1}{n} \sum_{i=1}^{n}\left\|w_{i j}\right\|_{q_{j}}^{q_{j}}\right)^{\frac{1}{q_{j}}} \\
& =\|\varphi(w)\|_{Y},
\end{aligned}
$$

where the inequality follows from the classical theorem on averages (HLP52, Theorem 16]) applied to each of the summands with the corresponding exponent $q_{j}$, for $1 \leq j \leq k$, respectively.

To prove the rightmost inequality, for each $j \in[k]$, let $i_{j} \in[n]$ be such that 


$$
\begin{aligned}
& \left\|w_{i_{j} j}\right\|_{q_{j}}=\max _{1 \leq i \leq n}\left\|w_{i j}\right\|_{q_{j}} \text {. Then } \\
& \begin{aligned}
\|\varphi(w)\|_{Y} & =\frac{1}{k} \sum_{j=1}^{k}\left(\frac{1}{n} \sum_{i=1}^{n}\left\|w_{i j}\right\|_{q_{j}}^{q_{j}}\right)^{\frac{1}{q_{j}}} \leq \frac{1}{k} \sum_{j=1}^{k}\left(\max _{1 \leq i \leq n}\left\|w_{i j}\right\|_{q_{j}}\right) \\
& =\frac{1}{k} \sum_{j=1}^{k}\left\|w_{i_{j} j}\right\|_{q_{j}} \leq \frac{1}{k} \sum_{j=1}^{k}\left(\sum_{l=1}^{k}\left\|w_{i_{j} l}\right\|_{q_{j}}\right) \\
& =\sum_{j=1}^{k}\left(\frac{1}{k} \sum_{l=1}^{k}\left\|w_{i_{j} l}\right\|_{q_{j}}\right)=\sum_{j=1}^{k}\left\|w_{i_{j}}\right\|_{Y} \\
& \leq k \max _{1 \leq i \leq n}\left\|w_{i}\right\|_{Y}=k\|w\|_{L_{\infty}^{n}(Y) .}
\end{aligned}
\end{aligned}
$$

Thus, (9) holds, and therefore, by (6),

$$
\frac{1}{C}\|w\|_{L_{\infty}^{n}(Y)} \leq\|\varphi(w)\|_{Y} \leq k\|w\|_{L_{\infty}^{n}(Y)}
$$

Acknowledgements: We would like to thank Assaf Naor for suggesting the problem, and Florence Lancien for valuable discussions.

The first named author was supported by the National Science Foundation under Grant Number DMS-1700176.

\section{References}

[AH11] S. A. Argyros, R. G. Haydon, A hereditarily indecomposable $\mathcal{L}_{\infty}$-space that solves the scalarplus-compact problem. Acta Math. 206 (2011), no. 1, 1-54.

[Beg99] B. Begun, A remark on almost extensions of Lipschitz functions, Israel J. Math., 109 (1999), 151-155.

[BP61] A. Benedek, R. Panzone, The space $L^{P}$, with mixed norm, Duke Math. J. 28 (1961), 301-324.

[BP60] C. Bessaga, A. Pełczyński, Banach spaces non-isomorphic to their Cartesian squares. I. Bull. Acad. Polon. Sci. Sér. Sci. Math. Astr. Phys. 8 (1960) 77-80.

[BBS02] A. Boccuto, A.V. Bukhvalov, A.R. Sambucini, Some inequalities in classical spaces with mixed norms, Positivity 6 (2002), no. 4, 393-411.

[Bou87] J. Bourgain, Remarks on the extension of Lipschitz maps defined on discrete sets and uniform homeomorphisms, in: Geometrical aspects of functional analysis (1985/86), 157-167, Lecture Notes in Math., 1267, Springer, Berlin, 1987.

[CS16] N. Clavero, J. Soria, Optimal rearrangement invariant Sobolev embeddings in mixed norm spaces. J. Geom. Anal. 26 (2016), no. 4, 2930-2954.

[DPS10] A. Defant, D. Popa, U. Schwarting, Coordinatewise multiple summing operators in Banach spaces, J. Funct. Anal. 259 (2010), no. 1, 220-242. 
[Die68] J. Diestel, An approach to the theory of Orlicz spaces of Lebesgue-Bochner measurable functions and to the theory of Orlicz spaces of finitely additive vector-valued set functions with applications to the representation of multilinear continuous operators. Ph.D. Thesis, The Catholic University of America. 1968. 60 pp.

[DU77] J. Diestel, J. J. Uhl, Vector measures. With a foreword by B. J. Pettis. Mathematical Surveys, No. 15. American Mathematical Society, Providence, R.I., 1977.

[FPP08] C. Fernández-González, C. Palazuelos, D. Pérez-García, The natural rearrangement invariant structure on tensor products. J. Math. Anal. Appl. 343 (2008), no. 1, 40-47.

[Fig72] T. Figiel, An example of infinite dimensional reflexive Banach space non-isomorphic to its Cartesian square. Studia Math. 42 (1972), 295-306.

[GJ73] D. P. Giesy, R. C. James, Uniformly non- $\ell^{(1)}$ and B-convex Banach spaces. Studia Math. 48 (1973), 61-69.

[GNS12] O. Giladi, A. Naor, G. Schechtman, Bourgain's discretization theorem, Annales Mathematiques de la faculte des sciences de Toulouse, vol. XXI (2012), no. 4, 817-837; (See also a later correction in arXiv:1110.5368v2.)

[GK03] G. Godefroy, N. J. Kalton, Lipschitz-free Banach spaces, Studia Math., 159 (2003), no. 1, 121-141.

[GS16] W. Grey, G. Sinnamon, The inclusion problem for mixed norm spaces. Trans. Amer. Math. Soc. 368 (2016), no. 12, 8715-8736.

[HLP52] G. H. Hardy, J. E. Littlewood, G. Pólya, Inequalities, Second Edition, Cambridge University Press, Cambridge, 1952.

[HM82] S. Heinrich, P. Mankiewicz, Applications of ultrapowers to the uniform and Lipschitz classification of Banach spaces, Studia Math., 73 (1982), no. 3, 225-251.

[HLN16] T. Hytönen, S. Li, A. Naor, Quantitative affine approximation for UMD targets, Discrete Analysis 2016(6):1-37, 2016.

[HN16+] T. Hytönen, A. Naor, Heat flow and quantitative differentiation, to appear in Journal of the European Mathematical Society; arXiv:1608.01915

[Jam50] R. C. James, Bases and reflexivity of Banach spaces. Ann. of Math. (2) 52, (1950). 518-527.

[JMS09] W. B. Johnson, B. Maurey, G. Schechtman, Non-linear factorization of linear operators. Bull. Lond. Math. Soc. 41 (2009), no. 4, 663-668.

[Lac74] H.E. Lacey, The isometric theory of classical Banach spaces. Die Grundlehren der mathematischen Wissenschaften, Band 208. Springer-Verlag, New York-Heidelberg, 1974.

[LN13] S. Li, A. Naor, Discretization and affine approximation in high dimensions. Israel J. Math. 197 (2013), no. 1, 107-129.

[Mat02] J. Matoušek, Lectures on discrete geometry. Graduate Texts in Mathematics, 212. SpringerVerlag, New York, 2002.

[Nao18] A. Naor, Metric dimension reduction: a snapshot of the Ribe program, Proc. Int. Cong. of Math. 2018, Rio de Janeiro, to appear; arXiv:1809.0237.

[NS07] A. Naor, G. Schechtman, Planar Earthmover is not in $L_{1}$, SIAM J. Computing, 37 (2007), $804-826$.

[Ost13] M. I. Ostrovskii, Metric Embeddings: Bilipschitz and Coarse Embeddings into Banach Spaces, de Gruyter Studies in Mathematics, 49. Walter de Gruyter \& Co., Berlin, 2013.

[Ray89] Y. Raynaud, Finite representability of $\ell_{p}(X)$ in Orlicz function spaces. Israel J. Math. 65 (1989), no. 2, 197-213.

[Rea90] C. J. Read, When $E$ and $E[E]$ are isomorphic, in: Geometry of Banach Spaces, Strobl, 1989, in: London Math. Soc. Lecture Note Ser., vol. 158, Cambridge Univ. Press, Cambridge, 1990, pp. $245-252$.

[Rib76] M. Ribe, On uniformly homeomorphic normed spaces, Ark. Mat., 14 (1976), no. 2, $237-244$. 
[Sem60] Z. Semadeni, Banach spaces non-isomorphic to their Cartesian squares. II. Bull. Acad. Polon. Sci. Sér. Sci. Math. Astr. Phys. 8 (1960) 81-84.

Department of Mathematics and Computer Science, St. John's University, 8000 Utopia PARKWAy, QueEns, NY 11439, USA

E-mail address: ostrovsm@stjohns.edu

Department of Mathematics, Miami University, Oxford, OH 45056, USA

E-mail address: randrib@miamioh.edu 